\title{
C-terminal amino acids are essential for human heat shock protein 70 dimerization
}

\author{
Guillaume Marcion • Renaud Seigneuric • Evelyne Chavanne - Yves Artur • \\ Loïc Briand • Tarik Hadi • Jessica Gobbo • Carmen Garrido • Fabrice Neiers
}

Received: 17 April 2014 / Revised: 3 July 2014 / Accepted: 4 July 2014 /Published online: 17 July 2014

(C) The Author(s) 2014. This article is published with open access at Springerlink.com

\begin{abstract}
The human inducible heat shock protein 70 (hHsp70), which is involved in several major pathologies, including neurodegenerative disorders and cancer, is a key molecular chaperone and contributes to the proper protein folding and maintenance of a large number of protein structures. Despite its role in disease, the current structural knowledge of hHsp70 is almost exclusively based on its Escherichia coli homolog, DnaK, even though these two proteins only share $\sim 50 \%$ amino acid identity. For the first time, we describe a complete heterologous production and purification strategy that allowed us to obtain a large amount of soluble, full-length, and non-tagged hHsp70. The protein displayed both an ATPase and a refolding activity when combined to the human Hsp40. Multi-angle light scattering and bio-layer
\end{abstract}

Guillaume Marcion, Renaud Seigneuric, Carmen Garrido, and Fabrice Neiers contributed equally to the work.

Electronic supplementary material The online version of this article (doi:10.1007/s12192-014-0526-3) contains supplementary material, which is available to authorized users.

G. Marcion $\cdot$ R. Seigneuric $(\bowtie) \cdot$ T. Hadi $\cdot$ J. Gobbo $\cdot$ C. Garrido INSERM, UMR 866, 7 blvd Jeanne d'Arc, 21000 Dijon, France e-mail: renaud.seigneuric@u-bourgogne.fr

G. Marcion • R. Seigneuric $\cdot$ E. Chavanne $\cdot$ Y. Artur $\cdot$ T. Hadi $\cdot$

$\mathrm{J}$. Gobbo $\cdot$ C. Garrido $\cdot$ F. Neiers

Université de Bourgogne, Esplanade Erasme, Dijon, France

E. Chavanne $\cdot$ Y. Artur $\cdot$ L. Briand $\cdot$ F. Neiers

Centre des Sciences du Goût et de l'Alimentation, INRA UMR 1324,

CNRS UMR 6265, Université de Bourgogne, Dijon, France

J. Gobbo $\cdot$ C. Garrido

Anticancer Center Georges François Leclerc, Dijon, France

F. Neiers $(\bowtie)$

CSGA, 17 rue Sully, 21000 Dijon, France

e-mail: fabrice.neiers@u-bourgogne.fr interferometry analyses demonstrated the ability of hHsp70 to homodimerize. The role of the C-terminal part of hHsp70 was identified and confirmed by a study of a truncated version of hHsp70 that could neither dimerize nor present refolding activity.

Keywords Hsp70 · HSPA1A - Dimer - Monomer - Cancer

$\begin{array}{ll}\text { Abbreviations } \\ \text { hHsp70 } & \text { human heat shock protein } 70 \\ \text { hHsp40 } & \text { human heat shock protein } 40 \\ \text { NBD } & \text { nucleotide binding domain } \\ \text { SBD } & \text { substrate binding domain } \\ \text { BLI } & \text { Bio-Layer Interferometry } \\ \text { SEC } & \text { size exclusion chromatography } \\ \text { SEC-MALS } & \begin{array}{l}\text { size exclusion chromatography } \\ \text { multi-angle light scattering }\end{array} \\ & \text { human heat shock cognate protein }\end{array}$

Introduction

Molecular chaperones assist in the non-covalent folding or unfolding of a polypeptide chain, refolding of misfolded intermediates, the assembly or disassembly of a complex, and its proper localization in the cell (Ellis 1987; Fink 1999; Taipale et al. 2010). Many cellular proteins tend to aggregate and become insoluble after a stress such as a heat shock, and the molecular chaperones that play a role in this process include members of the heat shock protein (Hsp) family. Hsps are structurally and functionally diverse proteins and are classified according to their approximate molecular mass. These proteins include six families: small Hsps, Hsp40, Hsp60, Hsp70, Hsp90, and Hsp110 (Brodsky and Chiosis 2006; Garrido et al. 2006; Mayer and Bukau 2005; Powers and Workman 2007; 
Schmitt et al. 2006). The human Hsp70 family is encoded by 13 genes members (excluding the pseudogenes) (Brocchieri et al. 2008). At the protein level, the constitutively-expressed Hsc70 and the inducible Hsp70 share nearly $80 \%$ amino acid sequence identity and differ by their C-terminal region (Dworniczak and Mirault 1987; Jinwal et al. 2013; Maeda et al. 2007). Present at low or undetectable levels in most unstressed normal cells and tissues, Hsp70 (also referred to as Hsp72, Hsp70-1, or HspA1A) is among the most stressinducible molecular chaperones (Dworniczak and Mirault 1987; Freeman et al. 1995). This $\sim 70 \mathrm{kDa}$ chaperone interacts with Hsp40 to accommodate its substrates, binds to short linear stretches of hydrophobic residues and refolds its substrate properly through cycles of ATP binding, hydrolysis, and release (Ellis et al. 2007; Schlecht et al. 2011; Taipale et al. 2010). Acting within the chaperone network, Hsp70 is central in protein folding and homeostasis (Bukau et al. 2000; Hartl et al. 2011; Hartl and Hayer-Hartl 2002). Human Hsp70 (hHsp70) is involved in several pathologies, including neurodegenerative disorders and cancer, where it exerts both a chaperone and an anti-apoptotic function (Garrido et al. 2001; Hartl et al. 2011; Jaattela 1999; Seigneuric et al. 2011b). The modulation of hHsp70 activity may thus represent an interesting therapeutic approach for some of these pathologies (Calderwood 2010, 2013; Gobbo et al. 2011; Jego et al. 2010; Seigneuric et al. 2011a; Stangl et al. 2011). Despite the importance and relevance of hHsp70 in health and disease, the most mechanistic information has been inferred from comparisons with its bacterial homolog, DnaK (Bhattacharya et al. 2009; Kityk et al. 2012; Kumar et al. 2011; Swain et al. 2007; Taipale et al. 2010; Vogel et al. 2006), despite the fact that human and bacterial Hsp70 share only $\sim 50 \%$ amino acid sequence identity (Hunt and Morimoto 1985; Nicolaï et al. 2013; Zhu et al. 1996). The hHsp70 is divided into two functional domains connected by a short linker: an amino-terminal nucleotide binding domain (NBD) and a carboxyl-terminal substrate binding domain (SBD). Allosteric regulation of hHsp70 between these two domains remains to be elucidated (Nicolaï et al. 2013). In addition, there are no full-length structures of the members of the hHsp70 protein family available, most likely due to the limited amount of pure available protein and/or technical challenges. Therefore, current structural representations of hHsp70 are based on the full-length structure of Escherichia coli DnaK solved by NMR (PDB ID: 2KHO) (Bertelsen et al. 2009). More recently, full-length $E$. coli DnaK structures in an ATP-bound state were solved using X-ray diffraction (PDB ID: 4B9Q, and 4JNE) (Kityk et al. 2012; Qi et al. 2013). Thus, most of the information on hHsp70 is derived from the $E$. coli Hsp70 homolog DnaK. A few independent studies have revealed a mixture of monomeric and dimeric forms after purification, but the in vivo relevance of these forms is a debatable point (Palleros et al. 1993; Richarme and Kohiyama 1993).
Using electron microscopy, Thompson et al. demonstrated that a DnaK mixture of dimer and monomer led to monomerization following substrate addition (Thompson et al. 2012). With regards to hHsp70, different groups reported the existence of oligomeric states from monomer to high order oligomers (Angelidis et al. 1999; Aprile et al. 2013; Nemoto et al. 2006). A variety of mechanisms have been proposed to explain the change of oligomeric state, including cystein oxidation-dependence (Nemoto et al. 2006) or temperature dependency (Angelidis et al. 1999). Recently, an interaction between the linker and the SBD has been proposed to mediate the oligomerization of hHsp70 (Aprile et al. 2013). Protein quantity is still a limiting step for biophysical or structural studies allowing for the characterization of the oligomeric state. To date, only commercial hHsp70 (HspA1A), sold by Stressgen, is available; therefore, the process of purification remains confidential. The heterologous expression and purification of the chaperone family proteins tends to exhibit low recovery and specificity (Nicoll et al. 2006). Some studies report a partial purification of hHsp70 fused to another protein (Macejak et al. 1990) or to a His-tagged protein (Aprile et al. 2013; Nemoto et al. 2006). Moreover, it has been demonstrated that small $\mathrm{N}$ - or C-termini extensions of the rat $\mathrm{Hsc} 70$ homolog or hHsp70 may alter their ATPase activities and peptide binding (Boice and Hightower 1997). In the present paper, a detailed methodology allowing for the production of a large quantity of a recombinant, pure, full-length, and fully active hHsp70 is described. This approach enabled us to explore, for the first time, the oligomeric state of a nontagged hHsp70 using an improved size exclusion chromatography (SEC) technology that can provide an absolute molecular mass: SEC-MALS (multi-angle light scattering). Using additional biophysical, and biochemical studies, we demonstrate the molecular basis driving the formation of the observed dimeric state.

\section{Results}

Characterization of the full-length hHsp70 and the delta-hHsp70

A synthetic, codon-optimized coding sequence for hHsp70 expression was introduced in a pET21a plasmid. Among the different cells that were tested for pET21-hHsp70 plasmid expression, BL21 Star (DE3) presented the highest level of expression. After transformation and production, the samples were analyzed by sodium dodecyl sulfate polyacrylamide gel electrophoresis (SDS-PAGE). Two bands were observed after induction: an intense band that migrated at approximately $70 \mathrm{kDa}$ (full-length hHsp70) and a second, less intense band that migrated at approximately $60 \mathrm{kDa}$ (delta-hHsp70) (Fig. 1a, lane 1). 
A

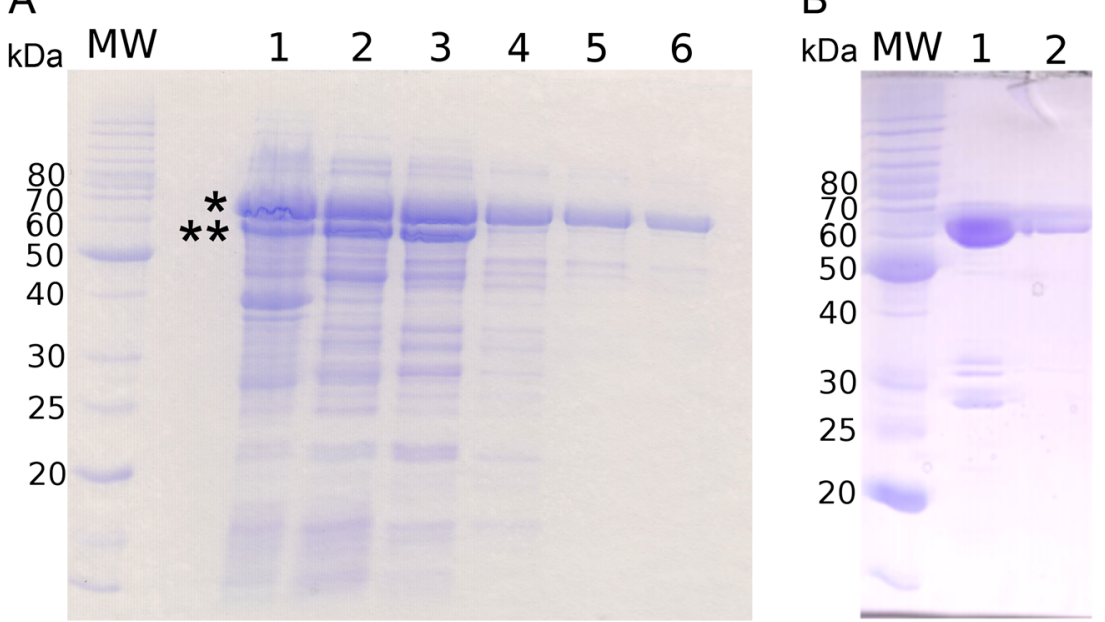

Fig. 1 Purification of full-length hHsp70 and delta-hHsp70. a Proteins were separated by $12 \%$ SDS-polyacrylamide gel electrophoresis and stained with Coomassie blue. The different lanes correspond to cell pellets $12 \mathrm{~h}$ after IPTG induction (lane 1), supernatant after sonication and centrifugation (lane 2), pool after the DEAE exchange column chromatography (lane 3), pool after the Q-sepharose exchange column chromatography (lane 4), pool after the Superdex 200 chromatography

Peptide mass fingerprinting, involving tryptic cleavage combined with MALDI-ToF analysis, confirmed that the $70 \mathrm{kDa}$ protein corresponded to the full-length hHsp70 (Supplemental figure $1 \mathrm{~A}$ and $\mathrm{C}$ ). All peptide fragments corresponded to hHsp70. This analysis also showed that the contamination from endogenous DnaK was non-detectable or absent. Moreover, these fragments covered the entire protein length.

During production, hHsp70 showed a sensitivity to a specific proteolysis, resulting in a band localized at approximately $60 \mathrm{kDa}$ (delta-hHsp70) (Fig. 1, panel a). This band was observed in all of the different production and purification batches tested, similar to other studies where Hsp70 was produced (Gross and Hessefort 1996; Peake et al. 1998). Mass spectroscopy fingerprinting analysis of this band showed a panel of peptides originating from hHsp70, thus proving that the pure protein obtained here corresponds to a truncated form of the hHsp70. The peptide analysis (Supplemental figure $1 \mathrm{~B}$ and D) revealed the presence of peptide fragments from the third amino acid residue. Moreover, none of the fragments were observed after the K561 amino acid residue, thereby suggesting a protein mass of $61.4 \mathrm{kDa}$ and a proteolysis cleavage occurring after the K561 amino acid residue.

\section{Purification of full-length hHsp70 and delta-hHsp70}

Protein purity after the different purification steps is shown in Fig. 1, panel a. After the centrifugation of sonicated cells, two thirds of the total recombinant hHsp70 was found in the pellet and one third was found in the (lane 5), and after the final Q-sepharose exchange column chromatography (lane 6). The star and double star indicate full-length hHsp70 and delta-hHsp70, respectively. b The pool of delta-hHsp70 after the DEAE exchange column chromatography (lane 1) and after purification on exclusion chromatography using a Superdex 200 (lane 2) was analyzed by SDS-PAGE. $M W$ molecular mass markers

supernatant. Different refolding strategies were carried out on the inclusion bodies without success because the resulting soluble protein showed no functional activity in the tested conditions (data not shown). The soluble hHsp70 obtained after the first centrifugation (supernatant) was loaded on an anion exchange resin column (DEAE resin). Interestingly, this step allowed us to separate the two hHsp70 forms observed after production. Delta-hHsp70 eluted at approximately $0.15 \mathrm{mM} \mathrm{KCl}$, whereas the fulllength hHsp70 eluted at $0.20 \mathrm{M} \mathrm{KCl}$. The latter value is in agreement with the low theoretical isoelectric $\mathrm{pH}$ of 5.5 calculated for the full-length hHsp70. This observation also suggests a higher value of the isoelectric $\mathrm{pH}$ for delta-hHsp70. A simulated truncation of hHsp70 leading to a $60 \mathrm{kDa}$ protein showed that only a C-terminal truncation of the protein increases the isoelectric $\mathrm{pH}$ by $0.3 \mathrm{pH}$ units, whereas an $\mathrm{N}$-terminal truncation decreases this value by $0.2 \mathrm{pH}$ units. These isoelectric $\mathrm{pH}$ differences between the two forms are also in agreement with the mass spectroscopy experiment, suggesting a truncation occurring after the $\mathrm{K} 561$ residue.

Fractions containing hHsp70 were pooled and loaded onto a strong anion exchange resin (Q-sepharose resin) followed by size exclusion chromatography (Superdex 200 Hiload 26/60). Next, the pooled fractions were reloaded on the strong anion exchange resin (Q-sepharose resin). Samples of each purification step were loaded on a $12.5 \%$ SDS-PAGE (Fig. 1, panel a). Thirty milligrams of pure hHsp70 was obtained per liter of culture, corresponding to a purification efficiency of approximately $15 \%$ of the total hHsp70 produced (soluble and nonsoluble). 
With regards to delta-hHsp70 separated after the first anion exchange resin column, this protein sample was concentrated to a $5-\mathrm{mL}$ final volume and loaded on a gel filtration column (Superdex 200 Hiload 26/60). Again, sample of each purification steps were loaded on a $12.5 \%$ SDS-PAGE (Fig. 1, panel b).

After 12 months, the full-length hHsp70 was again tested without signs of protein precipitation. Further, its folding activity was similar to the freshly produced protein, thereby suggesting that long-term storage did not alter the chaperone activity.

Intrinsic tryptophan fluorescence emission

To check the quality of the full-length hHsp70, we recorded its tryptophan (Trp) fluorescence emission spectrum before and after denaturation with two concentrations of guanidine chloride. To select a typical Trp fluorescence emission, a 295-nm excitation wavelength was used. Full-length hHsp70 contains two Trp (amino acid position 90 and 580). The maximum emission fluorescence signal due to the Trp90 and Trp580 amino acid residues at $349 \mathrm{~nm}$ supports their polar environment location (Fig. 2). After being exposed to a 2- and 4-M guanidine chloride solution, the full-length hHsp70 maximum emission spectra (at 354 and $356 \mathrm{~nm}$, respectively) presented a red shift compared to the non-denatured protein, typical of an increased solvent exposure (Fig. 2). The red shift and the observed maximum emission intensity decrease demonstrated a full-length hHsp70 denaturation. The observations support that the protein was initially properly folded in the starting solution before denaturation.

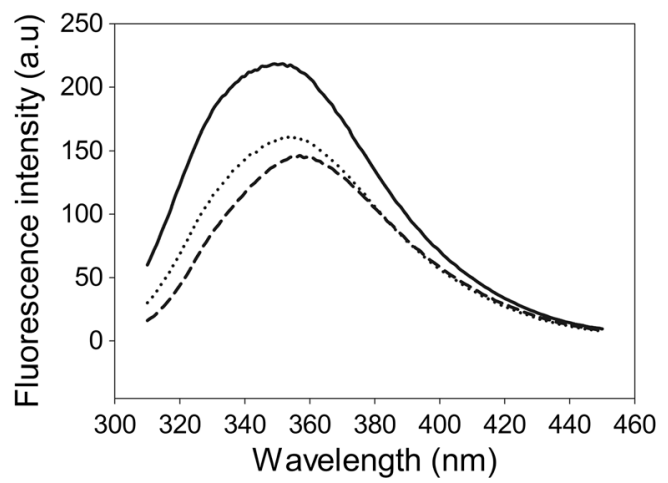

Fig. 2 Fluorescence emission spectra of full-length hHsp70 with or without denaturation. The spectra of $5 \mu \mathrm{M}$ protein were measured in $20 \mathrm{mM}$ Tris- $\mathrm{HCl} \mathrm{pH} 8.0$ buffer (solid curve), in the same buffer supplemented with $2 \mathrm{M}$ of guanidine chloride (dotted curve), or in $4 \mathrm{M}$ of guanidine chloride (dashed curve). After excitation at $295 \mathrm{~nm}$, the measured maximum emission spectra were 349, 354, and $356 \mathrm{~nm}$ (red shift), respectively, while the maximum of fluorescence emission spectra decreased. One representative curve is shown $(n=4)$
Secondary structure analysis of recombinant full-length hHsp70 and delta-hHsp70

To confirm the folding of full-length hHsp70 and deltahHsp70, we carried out circular dichroïsm spectrophotometer analysis. The recorded signals between 188 and $260 \mathrm{~nm}$ were superposed (Fig. 3). The good correlation of the two spectra supports a similar folding of the full-length and truncated hHsp70 forms. The far-UV spectra of the two proteins displayed a positive peak centered at $193 \mathrm{~nm}$ and two negative peaks at 210 and $218 \mathrm{~nm}$, showing the helical character of both proteins. The deconvolution of the circular dichroïsm (CD) spectra revealed that the full-length hHsp70 was composed of approximately 50-60\% alpha-helix and 25-30\% beta-sheet, whereas the truncated form revealed 40-50\% alpha-helix and $50 \%$ beta-sheet.

Oligomeric state analysis of recombinant full-length hHsp70 and delta-hHsp70

Using size exclusion chromatography (SEC) coupled to refraction index and multi-angle light scattering detectors (MALS), we determined the oligomeric state of full-length hHsp70 and delta-hHsp70. The three light scattering detectors exhibited a superposed signal characteristic of a monodisperse protein for both proteins. A single peak suggested homogenous samples in both cases (Fig. 4a, b). This last observation supports the notion that proteolysis occurs with a single cleavage resulting in delta-hHsp70. Moreover, the SEC-MALS spectra analysis showed a low percentage of aggregated forms (less than $1 \%$ ) for both proteins. This last observation supports the fact that the samples were properly folded and stable.

The recombinant full-length hHsp70 data analysis showed a single peak with a calculated mass from the MALS experiment of $139 \mathrm{kDa}$, corresponding to a hHsp70 dimer. The

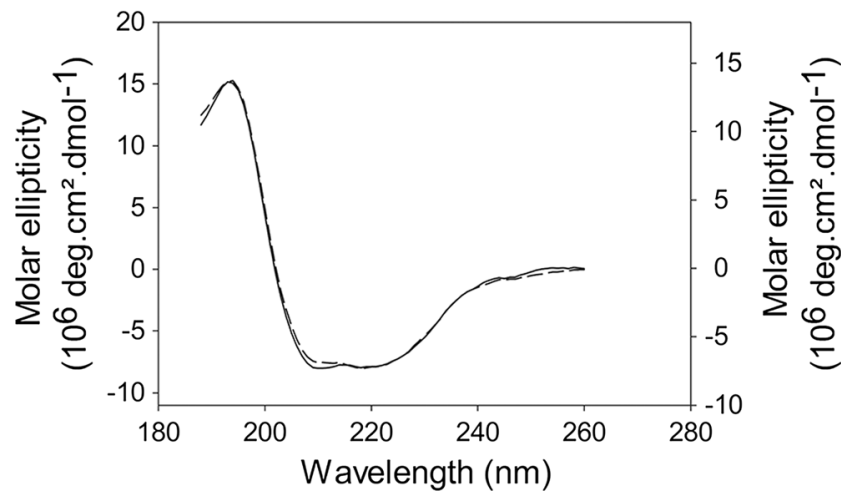

Fig. 3 Secondary structure analysis by circular dichroism. Spectra of the full-length hHsp70 (solid curve, left axis) and delta-hHsp70 (dashed curve, right axis) are presented in molar ellipticity $\left(\mathrm{deg} . \mathrm{cm}^{2} . \mathrm{dmol}{ }^{-1}\right)$. The spectra were recorded at $20{ }^{\circ} \mathrm{C}$ in a $0.01-\mathrm{cm}$ path cell length with a $5-\mu \mathrm{M}$ protein concentration in a $20-\mathrm{mM}$ Tris- $\mathrm{HCl} \mathrm{pH} 8.0$ buffer 

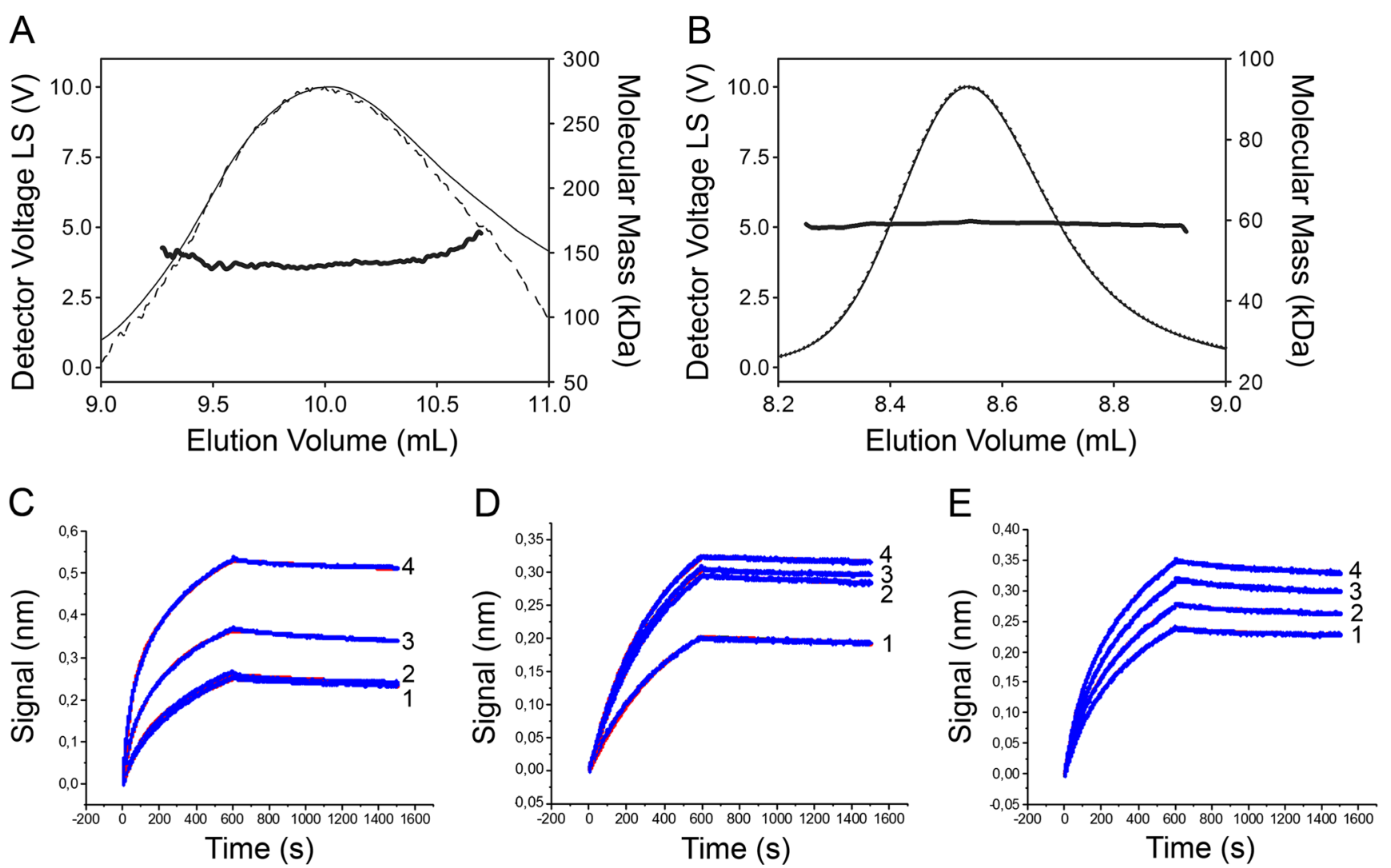

Fig. 4 Oligomeric state analysis of hHsp70. After size exclusion chromatography $(S E C)$ and separation with a suitable chromatography column (MP015S5 column from Wyatt for delta-hHsp70, and MP030S5 column from Wyatt for the full-length hHsp70), the molecular mass was determined from the Raleigh ratio, measured by static light scattering and the refractive index. The calculated molecular mass (bold black curve), refraction index (dotted black curve), and light scattering (black curve) are shown. The computed molecular masses are as follows: $139 \mathrm{kDa}$ (fulllength hHsp70: dimer) (a), and $59 \mathrm{kDa}$ (delta-hHsp70: monomer) (b). c,

theoretical mass for a dimer is estimated at $140 \mathrm{kDa}$ (Fig. 4a, b).

The molecular mass of delta-hHsp70 estimated by a MALS instrument was $59 \mathrm{kDa}$, thus suggesting a monomeric state of the protein. These data were in agreement with the results obtained by SDS-PAGE and mass spectroscopy fingerprinting, thereby suggesting the following: (i) a molecular mass of approximately $60 \mathrm{kDa}$ and (ii) the homogeneity of the sample supports a single cleavage site leading to truncated $\mathrm{hHsp} 70$. The absence of major structural modifications between the full-length protein in the dimeric state and the truncated form in the monomeric state, as demonstrated by circular dichroism, suggest no major structural changes during the dimerization process. To further demonstrate the existence of a hHsp70:hHsp70 interaction, we have quantified the following protein-protein interactions: (1) hHsp70:hHsp70, (2) hHsp70:hHsp40, and (3) DnaK:DnaK with another technique: the Bio-Layer Interferometry (BLI). BLI is an optical- and label-free technique sensitive to an increase of mass bound to a biosensor (e.g., bound analyte). BLI provides sensorgrams d, and e Sensorgrams were obtained by Bio-Layer Interferometry $(B L I)$. The different concentrations of the analytes in PBS are: panel $\mathbf{c}$ hHsp70 (1: 200, 2: 300, 3: 400, and 4: $500 \mathrm{nM})$; panel d hHsp40 (1: 20, 2: 30, 3: 40, and 4: $50 \mathrm{nM}$ ); and panel e DnaK (1: 200, 2: 300, 3: 400 and 4: $500 \mathrm{nM}$ ). The best fit is provided with the 1:1 model (red fit) with a $\mathrm{K}_{\mathrm{D}}$ value of $4.5+/-0.1 \mathrm{nM}, \mathrm{a} \mathrm{r}^{2}$ of 0.99 for hHsp70:hHsp70 (c), a $\mathrm{K}_{\mathrm{D}}$ value of $0.52+/-0.01 \mathrm{nM}$, a $\mathrm{r}^{2}$ of 0.99 for hHsp70:hHsp40 (d), and a $\mathrm{K}_{\mathrm{D}}$ value of $5.4+/-0.2 \mathrm{nM}$, a r $^{2}$ of 0.99 for DnaK:DnaK (e)

like surface plasmon resonance. To perform the experiment, after biotynilation of $\mathrm{hHsp} 70$, the protein was immobilized onto the biosensor tip. Then, we tested the ability of hHsp70 to interact with hHsp70 in solution. These results clearly demonstrate that hHsp70 is interacting with hHsp70 in solution, demonstrating the capacity of $\mathrm{hHsp} 70$ to dimerize. The technique allows observing the small fraction of dimer exchanging their partner (supported by the 1:1 model fitting the sensograms). Moreover, hHsp70 presents a high-affinity in the low nanomolar range for itself in agreement with the ranges observed for dimers $\left(K_{D} \sim 5 \mathrm{nM}\right.$, Fig. $\left.4 \mathrm{c}\right)$. We also show that, the well-known hHsp70:hHsp40 interaction has a greater affinity $\left(K_{D} \sim 0.5 \mathrm{nM}\right.$, Fig. $\left.4 \mathrm{~d}\right)$. Also, in line with the literature collated in Table 1, we confirm the DnaK:DnaK interaction (Fig. 4e), as a positive control of this experiment. Its affinity compares to the hHsp70:hHsp70 interaction $\left(K_{D} \sim 5 \mathrm{nM}\right)$.

In another BLI experiment, we used a previously selected peptide aptamer (A17) arising from a screen for its ability to bind to the N-terminal domain of hHsp70 (Rerole et al. 2011). As shown in Fig. 5, the measured signal shows a binding of 
Table 1 Studies detailing the oligomerization status of different Hsp70 family members along with the method used to determine the status

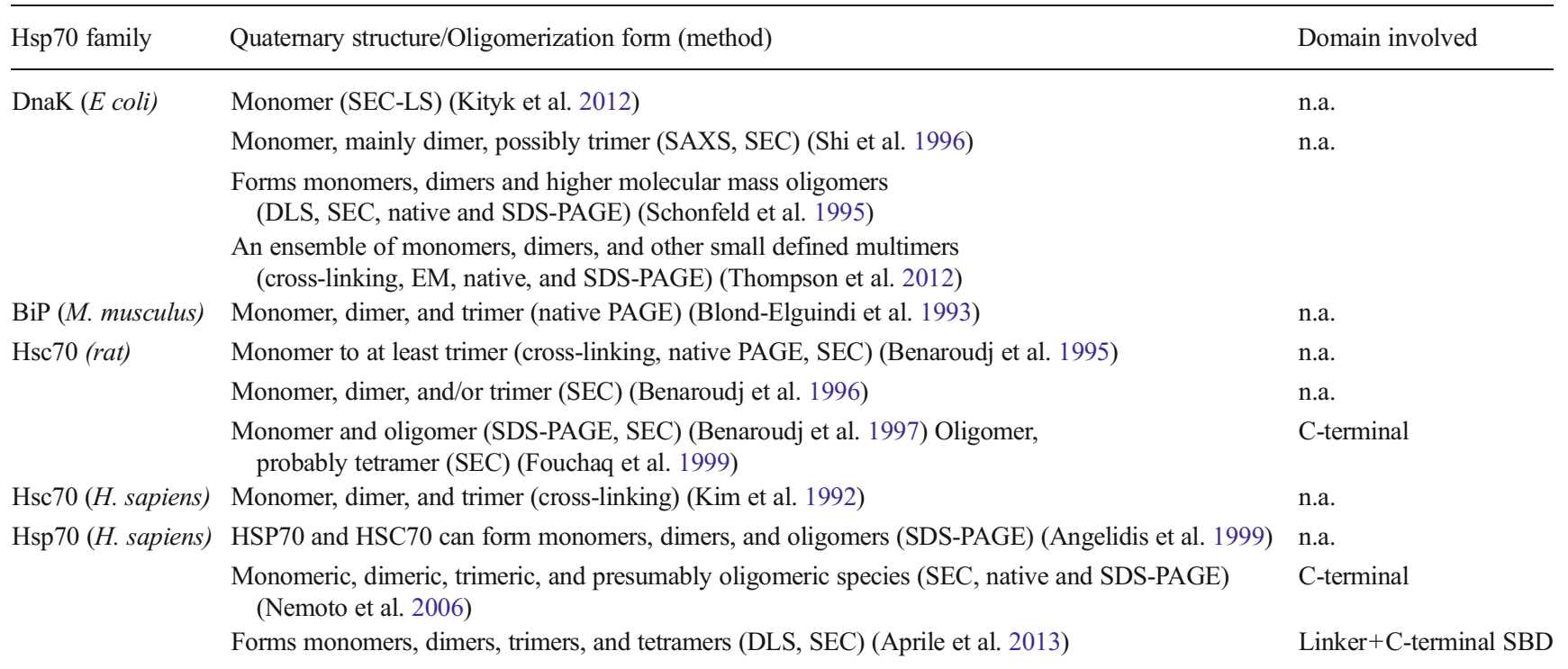

$D L S$ dynamic light scattering, $E M$ electron microscopy, $L S$ light scattering, $P A G E$ polyacrylamide gel electrophoresis, $S A X S$ small angle x-ray scattering, $S B D$ substrate binding domain, $S D S$ sodium dodecyl sulfate, $S E C$ size exclusion chromatography, n.a. not available

hHsp70 with the A17 peptide aptamer. This binding is only fit by a 1:2 (A17: hHsp70×2) binding model with a $K_{D}$ in the low $\mathrm{nM}$ range. It corresponds to the affinity of the hHsp70 (as a homodimer) for the A17 peptide aptamer. This result supports that the protein is properly folded. Also, it indirectly shows, by this third method, the dimeric state of the protein. Interestingly, in contrast to the A17:hHsp70 interaction, the A17:DnaK interaction is quite weak (around $6 \mathrm{nM}$ compared to $50 \mathrm{nM}$, respectively, Fig. 5). This further demonstrates the specificity of the A17 ligand for hHsp70.

\section{Functional assay}

Two aspects of hHsp70 function, ATPase activity and refolding activity were monitored.

ATPase activity was assessed by a homogeneous timeresolved fluorescence technique (HTRF) that follows the percentage of ATP to ADP enzymatic hydrolysis. The bar diagram represents the percentage of converted ATP (Fig. 6a): with no proteins (NP), with native firefly luciferase (NL), or with denatured firefly luciferase (DL) in a refolding buffer containing either no Hsps, our full-length hHsp70 alone, or with hHsp40. This data demonstrates the ATPase activity of hHsp70 compared to hHsp40. Moreover, this activity was dramatically increased in combination with its natural cochaperone, hHsp40. Adding a native client protein increased this ATPase activity that was further increased when the client protein is denatured. This result demonstrates the active state of the purified protein and its efficient coupling to human Hsp40.

The refolding activity of the recombinant full-length hHsp70 and delta-hHsp70 proteins was then measured by their ability to refold a denatured firefly luciferase protein. In this colorimetric assay, different types of $\mathrm{Hsp} 70$ are combined, one at a time, with a human Hsp40. The refolding percentage is compared to the positive (non-denatured, thus luminescent firefly luciferase) and negative (denatured, thus non-

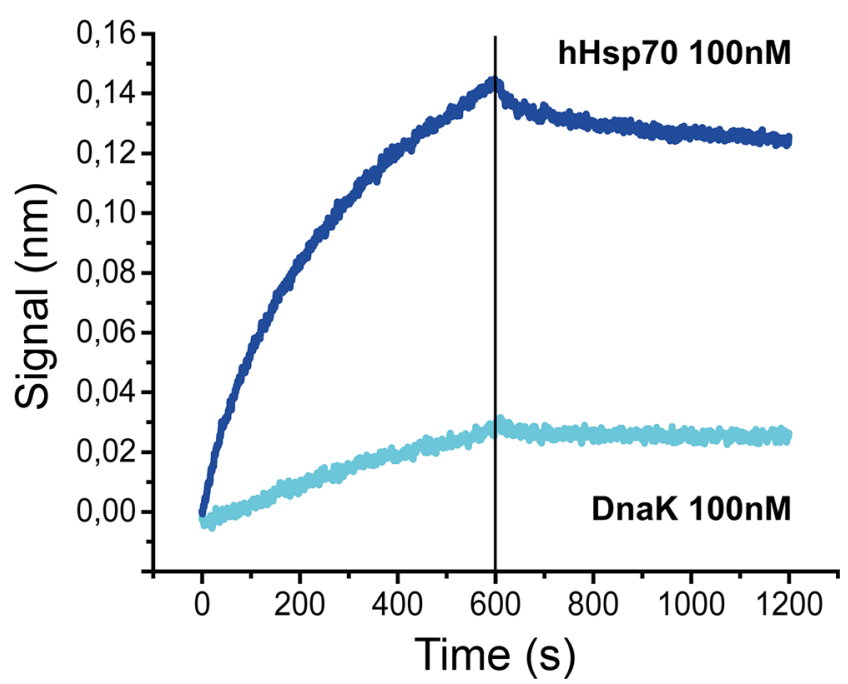

Fig. 5 Sensorgrams representing the interaction between $\mathrm{hHsp} 70$ and the Hsp70 peptide aptamer A17. In contrast to the capture of hHsp70 at $100 \mathrm{nM}$ with peptide aptamer A17 (dark blue sensorgram), the bottom sensorgram obtained by Bio-Layer Interferometry (BLI) (light blue) indicates that A17 and DnaK hardly interact. This stresses the actual differences in the $\mathrm{N}$-terminal region of $\mathrm{hHsp} 70$ and DnaK, although this region is largely considered as well conserved. A17:DnaK binding fits with a 1:1 model (not excluding a 1:2 model) and A17:hHsp70 with 1:2 model (excluding the simplest 1:1 model). An estimation of the $\mathrm{K}_{\mathrm{D}}$ is in the $50 \mathrm{nM}$ range for $A 17:$ DnaK whereas an estimation of the $K_{D}$ of A17:hHsp70 is around $6 \mathrm{nM}$ 
luminescent firefly luciferase, without Hsp70) controls. In order to monitor refolding following denaturation, different kinetics (60 $\mathrm{min}, 120 \mathrm{~min}, 180 \mathrm{~min}$ ) were investigated (data not shown). The best condition was obtained after a $180 \mathrm{~min}$ of refolding kinetics and used for all conditions. The Pareto plot (bar diagram in descending order) in Fig. 6b indicates that the full-length and non-tagged hHsp70 produced here presented a refolding activity when combined with Hsp40. The produced hHsp70 presented a refolding efficiency of $95 \%$. Interestingly, this activity was twofold higher compared to the commercially available hHsp70 (tagged). The delta-hHsp70 did not present any refolding activity. The lack of activity for this properly folded truncated protein can be explained by (i) the loss of dimerization ability, or (ii) the loss of an essential part of the protein for its chaperone activity, or (iii) both. As expected, the negative control presented a very low activity. In these conditions, this assay demonstrates the refolding activity (95\%) of the produced hHsp70.

\section{Discussion}

\section{Validation of hHSP70 structure}

In this paper, we report that hHsp70 can be expressed in milligram quantities using E. coli. The decrease of the fluorescence signal and the red shift of the emission frequency when a chaotropic agent is mixed with hHsp70 in solution

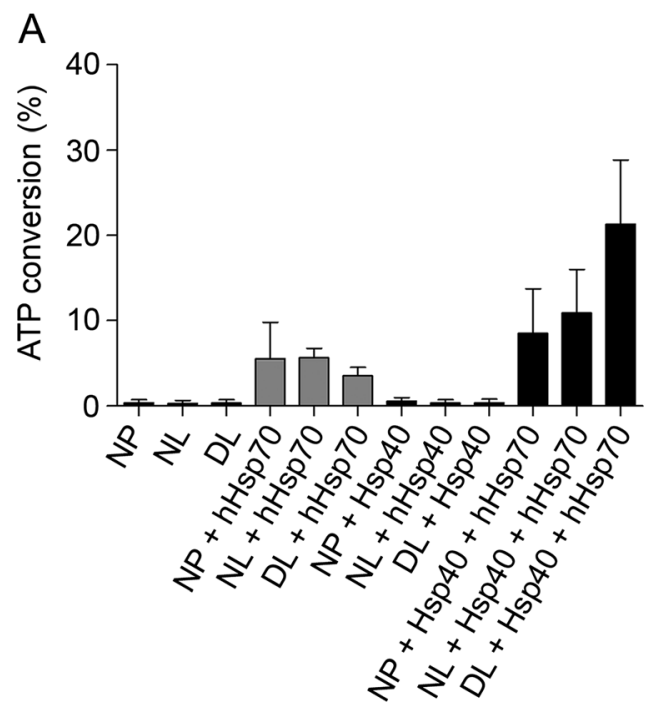

Fig. 6 Confirmation of the chaperone function with a firefly luciferase refolding assay and ATPase activity assay. The bar diagram represents the percentage of ATP to ADP hydrolysis (a): with no proteins $(N P)$, with native firefly luciferase $(N L)$, or with denatured firefly luciferase $(D L)$ in a refolding buffer containing either no Hsps, our full-length $\mathrm{hHsp} 70$ alone, hHsp40 alone, or the both Hsps. ADP formation was assessed by measuring the loss of d2-ADP fluorescence at $665 \mathrm{~nm}$, reported by cryptate fluorescence at $620 \mathrm{~nm}$ after $1 \mathrm{~h}$ of incubation. The percentage of ATP to ADP hydrolysis values are obtained from an ATP/ADP standard curve. support the quality of the purification. These modifications of the fluorescence are typically associated with the denaturation of a protein that was initially well-folded. The quantity of purified protein allows for the characterization of the secondary structures by the deconvolution of the $\mathrm{CD}$ spectra; the percentage of alpha-helix was estimated for the entire protein to be approximately $50-60 \%$ and the percentage of beta-sheet to be approximately $25-30 \%$. The percentage of alpha-helix and beta-sheet estimated from the crystal structure of the homolog E. coli DnaK are 36 and $27 \%$, respectively (PDB ID: 2KHO) (Bertelsen et al. 2009). The proportion of secondary structure elements in hHsp70 and in E. coli DnaK are thus in the same range. Our experimental data for hHsp70 supports the previous model prediction of the secondary structure of hHsp70 (Nicolaï et al. 2013). Sparse evidences of the oligomerization status of some Hsp70 family members are collated in Table 1. In this study, the SEC-MALS analysis revealed a monodisperse homodimeric hHsp70 with a very low percent of aggregated forms. The BLI experiments clearly confirm with another approach the dimeric state of the protein and provides us with a $K_{D}$ in the low nanomolar range $\left(K_{D} \sim 5 \mathrm{nM}\right)$ for the interaction between the two hHsp70 proteins forming the dimer.

Validation of hHsp70 activity

The activity of the purified protein was tested with a refolding assay using firefly luciferase. The observed activity was 2 to 3

B

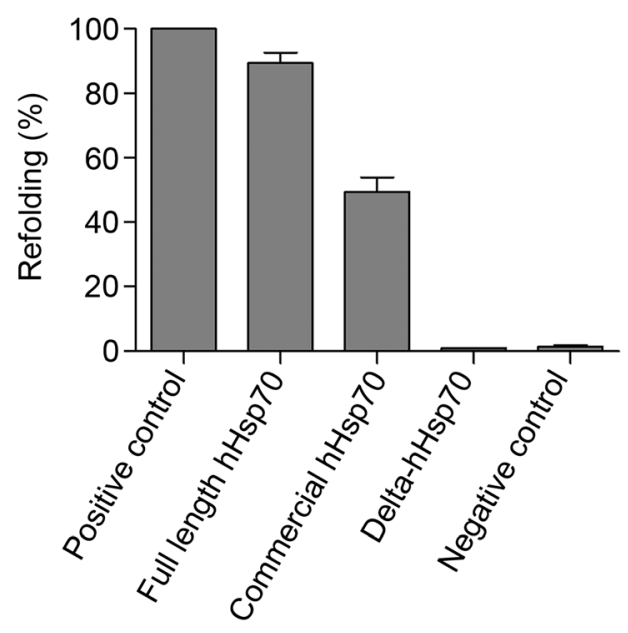

The Pareto plot represents the percentage of firefly luciferase refolding (b) in the presence of the full-length hHsp70, delta-hHsp70 (detailed in the present paper), and a commercially available hHsp70. The refolding of luciferase after denaturation $(0.2 \mathrm{M}$ guanidinium- $\mathrm{HCl}$ for $30 \mathrm{~min}$ at $25^{\circ} \mathrm{C}$ ) was measured by collecting total light at $560 \mathrm{~nm}$ after $3 \mathrm{~h}$ of incubation at $35{ }^{\circ} \mathrm{C}$. Values are compared to a positive control (nondenatured luciferase only, set to $100 \%$ of refolding) and a negative control (denatured luciferase incubated without Hsps) 
times higher compared to the commercially available Histagged $\mathrm{hHsp} 70$. This result is consistent with a previous observation of activity alteration when hHsp70 is tagged (Boice and Hightower 1997). The present results demonstrate that the hHsp70 produced here adopts its full native state and is fully functional as shown by its chaperone as well as its ATPase activity.

\section{C-terminal part involved in hHsp70 dimerization}

The BLI data, measuring the binding of hHsp70 with the A17 ligand (Fig. 5), demonstrates a high-affinity as the A17: hHsp70 interaction is around $6 \mathrm{nM}$. Besides, the comparison of all available model fittings is consistent with the homodimeric nature of $\mathrm{hHsp} 70$. This third proof is indirect but complementary to the two first evidences provided by the SECMALS data and the BLI determination of hHsp70: hHsp70.

Interestingly, we used a previously selected peptide aptamer (A17) for its ability to specifically bind to the Nterminal domain of hHsp70, further supporting the role of the C-terminal part in the dimerization. Although the N-terminal domain is generally considered to be the most conserved (compared to the C-terminal domain), our data enlightens actual differences between human and bacterial Hsp70 Nterminal regions (Aprile et al. 2013; Flaherty et al. 1990; Hartl and Hayer-Hartl 2009; Schmid et al. 1994; Zhang and Walker 1998).

Our peptide mass fingerprinting of the purified truncated form combined with the SEC-MALS analysis demonstrates the role of the C-terminal part in the dimerization (I562 to D641). Interestingly, this protease sensitive region has been previously reported for bovine Hsc70 (Chappell et al. 1987). Our observations of another Hsp70 family member raise the question of the physiological relevance of the truncation we present here for hHsp70 (cleavage site databases such as PeptideCutter and MEROPS did not allow us to identify a highly sequence-specific protease). Interestingly, deltahHsp70 does not present chaperone activity. This observation can be explained by the truncation of the C-terminal part containing the EEVD motif. The EEVD motif has been demonstrated to be necessary for the interaction with the folding partner Hsp40 (Freeman et al. 1995). The C-terminal part identified in the homodimerization present two cysteine residues. The full-length hHsp70 was observed at the same apparent mass by SDS-PAGE when loading with or without reductant dithiothreitol treatment (data not shown). Recently, a study suggested the role of the interaction between the interdomain linker and the SBD in the oligomerization process (Aprile et al. 2013). This result together with our SDSPAGE observations is in contrast with a previous study showing a disulfide bridge formed by cysteine 574 in the hHsp70 dimer (Nemoto et al. 2006). The last $80 \mathrm{C}$-terminal residues have been demonstrated to be involved in dimerization.
Interestingly, the sequence identity of these $80 \mathrm{C}$-terminal amino acids with DnaK is approximately $20 \%$, whereas the global identity is calculated at $50 \%$. This observation warns us to be careful in generalizing the results for DnaK as it presents an EEVKD sequence, whereas hHsp70 bears the EEVD motif.

\section{Conclusion}

In conclusion, this study provides strong evidence for the homodimeric nature of hHsp70 and identifies the last $80 \mathrm{C}$ terminal residues as involved in the dimerization process. Our experiments revealed that the dimerization process is governed by non-covalent interactions. As shown in the present paper, it is possible to purify large quantities of full-length non-tagged properly folded and active hHsp70, thereby opening up the possibility of the biophysical/biochemical studies presented in this study or radiocrystallography.

Although the physiological role of the hHsp70 homodimer remains to be elucidated, its dimeric nature may be a way to regulate its function, and potentially sets a new paradigm for human Hsp70.

\section{Materials and methods}

Construction of the hHsp70 expression plasmid

A codon-optimized gene encoding for hHsp70 expression in E. coli was synthesized (Geneart, Carlsbad, California). Nde1 and Sac1 restriction sites were inserted into the 5' and 3' ends, respectively, of the open reading frame coding sequence for hHsp70. The digested sequences were ligated in a pET21a plasmid previously opened by the same restriction enzymes in the cloning cassette. The resulting expression vector (pET21hHsp70) encodes a full-length hHsp70 translated in the following sequence:

MAKAAAIGIDLGTTYSCVGVFQHGKVEIIANDQGNRTTPSYVAFTDTERLIGDAAKNQVALNPQNTVFDA KRLIGRKFGDPVVQSDMKHWPFQVINDGDKPKV QVSYKGDTKAFYPEEISSMVLTKMKEIAEAYLGYPVT NAVITVPAYFNDSQRQATKDAGVIAGLNVLRIINEP TAAAIAYGLDRTGKGERNVLIFDLGGGTFDVSILTIDD GIFEVKATAGDTHLGGEDFDNRLVNHFVEEFKR KHKKDISQNKRAVRRLRTACERAKRTLSSSTQASLEI DSLFEGIDFYTSITRARFEELCSDLFRSTLEPVEKALR DAKLDKAQIHDLVLVGGSTRIPKVQKLLQDFFNGRD LNKSINPDEAVAYGAAVQAAILMGDKSENVQDLLLL DVAPLSLGLETAGGVMTALIKRNSTIPTKQTQIFTTYS DNQPGVLIQVYEGERAMTKDNNLLGRFELSGI PPAPRGVPQIEVTFDIDANGILNVTATDKSTGKANKIT 
ITNDKGRLSKEEIERMVQEAEKYKAEDEVQRERVSA KNALESYAFNMKSAVEDEGLKGKISEADKKKVLDKC QEVISWLDANTLAEKDEFEHKRKELEQVCNPIISGLY QGAGGPGPGGF GAQGPKGGSGSGPTIEEVD

The integrity of the construct was verified by DNA sequencing.

\section{Production of hHsp70}

BL21 Star ${ }^{\mathrm{TM}}$ (DE3), cells were transformed with the pET21hHsp70 plasmid. The selected clones obtained on agar Luria broth (LB) plate supplemented with ampicillin $(100 \mu \mathrm{g} / \mathrm{L})$ after cell transformation were used to inoculate a $100-\mathrm{mL}$ LB culture (100 $\mu \mathrm{g} / \mathrm{L}$ ampicillin). After $12 \mathrm{~h}, 20 \mathrm{~mL}$ of culture was transferred to $1 \mathrm{~L}$ of LB culture $(100 \mu \mathrm{g} / \mathrm{L}$ ampicillin $)$ and incubated at $37^{\circ} \mathrm{C}$. Cell growth was stopped by centrifugation at $4,000 \times \mathrm{g}, 20 \mathrm{~h}$ after induction by $1 \mathrm{mM}$ (final concentration) IPTG.

\section{Purification of hHsp70}

After production and centrifugation, cells were resuspended in buffer A $(50 \mathrm{mM}$ Tris- $\mathrm{HCl}, \mathrm{pH}$ 8.0) supplemented with $20 \mu \mathrm{M}$ of dithiothreitol. Cells were then disrupted by sonication at $4{ }^{\circ} \mathrm{C}$ and centrifuged at $17,000 \times g$ for 45 min at $4{ }^{\circ} \mathrm{C}$. The supernatant cell lysate was loaded onto an 80 -mL DEAESepharose column (GE Healthcare). After washing with two column volumes of buffer $\mathrm{A}$, proteins were eluted by a $\mathrm{KCl}$ linear gradient (0 to $1 \mathrm{M} \mathrm{KCl}$ in buffer A). A $40-\mathrm{mL}$ QSepharose column and a Superdex 200 Hiload 26/60 (GE healthcare, Little Chalfont, Buckinghamshire, UK) coupled to an AKTA FPLC were used for the following purification steps. Identical buffer, washing, and elution protocols were used on the Q-Sepharose and the DEAE column. Separation on the Superdex 200 was performed with an isocratic flow of buffer A. The protein concentration before size exclusion chromatography and after the last purification step was conducted using an Amicon Ultra (Millipore) filter unit with a cutoff of $10 \mathrm{kDa}$. The final pure protein fractions were pooled and concentrated to approximately $100 \mu \mathrm{M}$ and either directly frozen at $20^{\circ} \mathrm{C}$ or tested for activity. Purity of the proteins was assessed using SDS-PAGE with Coomassie-blue staining. Quantification of the full-length hHsp70 was determined using UV spectroscopy with a molar extinction coefficient of $33,350 \mathrm{M}^{-1} \mathrm{~cm}^{-1}$ at $280 \mathrm{~nm}$, calculated from the amino acid composition of the protein on ProtParam (http://web.expasy. org/protparam/). The same method was used to determine a molar extinction coefficient of $26,360 \mathrm{M}^{-1} \mathrm{~cm}^{-1}$ at $280 \mathrm{~nm}$, based on the mass spectroscopy protein length estimation. The theoretical isoelectric $\mathrm{pH}$ of the two forms was calculated using the ProtParam tool (http://web.expasy.org/protparam/). The purified and concentrated proteins at $10 \mu \mathrm{M}$ were frozen at $20^{\circ} \mathrm{C}$ in a $50-\mathrm{mM}$ Tris- $\mathrm{HCl} \mathrm{pH} 8.0$ buffer.
Intrinsic fluorescence measurement

Intrinsic fluorescence was measured using a Cary Eclipse spectrofluorimeter (Varian Instruments) equipped with magnetic stirrers and a Peltier temperature control unit. All experiments were conducted at $25{ }^{\circ} \mathrm{C}$. The emission spectra were recorded between 310 and $450 \mathrm{~nm}$ after excitation at $295 \mathrm{~nm}$. Both excitation and emission bandwidths were $5 \mathrm{~nm}$. The spectra were recorded in a $2-\mathrm{mL}$ cuvette of $1 \mathrm{~cm}$ path-length with a $5-\mu \mathrm{M}$ protein concentration in buffer $\mathrm{A}$ or buffer A with 2 and $4 \mathrm{M}$ guanidine chloride.

\section{Far-UV circular dichroism}

Far-UV circular dichroïsm (CD) spectra were recorded using a JASCO J-815 spectropolarimeter equipped with a Peltier temperature control set at $25{ }^{\circ} \mathrm{C}$. Using a $0.01-\mathrm{cm}$ path-length quartz cell (Hellma), the protein samples spectra $(5 \mu \mathrm{M}$ in $20 \mathrm{mM}$ Tris-HCl pH 8.0) were recorded with a scan speed of $50 \mathrm{~nm} \cdot \mathrm{min}^{-1}$ between 188 and $260 \mathrm{~nm}$. Spectra were averaged over 10 scans and corrected by subtraction of the spectra acquired for buffer (20 mM Tris- $\mathrm{HCl}, \mathrm{pH}$ 8.0) alone. $\mathrm{CD}$ measurements were normalized to protein concentration and presented as molar ellipticity deg. $\mathrm{cm}^{2} . \mathrm{dmol}^{-1}$ using the Jasco Spectra Analysis software. The spectra were decomposed to $\alpha$-helix, $\beta$-sheet, $\beta$-turn, and random coil elements using Yang's reference set (Venyaminov et al. 1993).

\section{Peptide mass fingerprinting}

Purified protein band sequences were identified by peptide mass fingerprinting using tryptic cleavage combined with MALDI-ToF analysis. After purification, the full-length or the truncated forms of hHsp70 were loaded on SDS-PAGE. The corresponding bands were cut and extracted from the gel. After reduction and alkylation, they were digested with $100 \mathrm{ng}$ of pig trypsin (PROMEGA). The peptides were injected on a LC-MS orbitrap. The PAPPSO platform (INRA, Jouy-enJosas) was employed to conduct the protein identification using MS-Fit (Protein Prospector, UCSF).

Size exclusion chromatography with on-line multi-angle light scattering (SEC-MALS)

The investigation of protein oligomeric states was performed on a Jasco PU-2080 Plus system consisting of a pump, a vacuum degasser, an autosampler, and a silica column MP015S5 or MP030S5 $(7.8 \mathrm{~mm}, 300 \mathrm{~mm}$ ), (Wyatt, Santa Barbara, CA). The MP015S5 column was used for the hHsp70 monomer, whereas the MP030S5 column was used for the hHsp70 dimer. The detection was carried out using a refractometer (RID-10A, Shimadzu) and a miniDAWN TREOS light scattering detector (Wyatt Technology). The 
column temperature was regulated at $20^{\circ} \mathrm{C}$. The buffer used for the isocratic gradient at $0.5 \mathrm{~mL} / \mathrm{min}$ was a $0.1-\mathrm{M}$ sodium phosphate buffer at $\mathrm{pH}$ 7.0. The $90^{\circ}$ detector of the miniDAWN TREOS was calibrated using a toluene solution, the other detectors $\left(45^{\circ}\right.$ and $\left.135^{\circ}\right)$ were normalized using a 10$\mathrm{mg} / \mathrm{mL}$ BSA solution in $0.1 \mathrm{M}$ phosphate buffer, $\mathrm{pH}$ 7.0. The toluene calibration of the system was confirmed for different molecular sizes and oligomeric states of known proteins.

The solute concentration (C) was obtained directly from the refractive index signal for each volume slice according to $\mathrm{C}=(\mathrm{Vi} \mathrm{K}) /(\mathrm{dn} / \mathrm{dc})$, where $\mathrm{Vi}$ is the detector output voltages for volume slice $\mathrm{i}$, and $\mathrm{K}$ is the calibration constant. $\mathrm{A} \mathrm{dn} / \mathrm{dc}$ value of $0.186 \mathrm{~mL} / \mathrm{g}$ was used for the calculations. The molecular mass of the protein was computed using the ASTRA V software (Wyatt Technology, Santa Barbara, CA, USA) based on the light scattering and refractive index signals.

\section{Bio-Layer Interferometry}

Bio-layer interferometry (BLI), an optical and label-free technique sensitive to an increase of mass bound to the biosensor (e.g., bound analyte), provides sensorgrams as surface plasmon resonance. The first BLI experiment (Fig. 4), measures the affinity of the interacting protein. In a first step, the proteins $(5 \mu \mathrm{g}$ of biotinylated $\mathrm{hHsp} 70$ or DnaK) were incubated in a PBS buffer with a 1:3 ratio molar ratio of biotin. The free biotin was removed using a desalting column (Pierce). Then, the biotinylated protein was immobilized onto streptavidin biosensor tips and dipped into wells containing the analyte at different concentrations in PBS: hHsp70 (200, $300,400$, and $500 \mathrm{nM})$; hHsp40 (20, 30, 40, and $50 \mathrm{nM})$; and DnaK (200, 300, 400, and $500 \mathrm{nM})$. The second BLI experiment (Fig. 5) allowed the measurement of the dimeric hHsp70 interaction with a specific ligand. A17 binds to the N-terminal region of hHsp70 (Rerole et al. 2011; Seigneuric et al. 2011a) and was biotinylated with a 1:3 ratio (biotinPEG4-NHS from Pierce EZ kit, prepared following the manufacturer's instructions). The free biotin was removed using a desalting column (Pierce), then, the biotinylated ligand (A17) was immobilized onto streptavidin biosensor tips and dipped into wells containing the analyte (hHsp70 or DnaK at $100 \mathrm{nM}$ in PBS). Both experiments (total volume in each well: $200 \mu \mathrm{L}$; shake speed: 1,000 rpm, association phase: $600 \mathrm{~s}$; dissociation phase: $600 \mathrm{~s}$ ) were background corrected, smoothed with the Savitzky-Golay algorithm and analyzed using OctetRED instrument software (ForteBio Data Analysis version 7.1.). For the first experiment (Fig. 4), all sensograms were fit with a 1:1 model. For the second type of experiment (Fig. 5), all available models were tested; but only the 1:2 model (bivalent analyte) was matching for the A17:hHsp70 interaction (excluding a 1:1 model). However, for the A17:DnaK interaction, the 1:1 model was matching (not excluding a more complex 1:2 model).
hHsp70 ATPase activity assay

Firefly luciferase was denatured with $6 \mathrm{M}$ guanidium-HCl, and then incubated with ATP $(100 \mu \mathrm{M})$ and/or Hsp40 $(160 \mathrm{nM})$ and/or hHsp70 $(800 \mathrm{nM})$ for an hour. ATP to ADP conversion was monitored using the HTRF transcreener ADP (Cisbio assays, Codolet, France), according to the manufacturer's instruction. Briefly, after refolding, $10 \mu \mathrm{L}$ of refolding mix was incubated for $1 \mathrm{~h}$ with $37.5 \mu \mathrm{L} 1 \mathrm{X}$ HTRF transcreener ADP enzymatic buffer, $5 \mu \mathrm{L}$ of Anti-ADP antibody coupled to the HTRF donor $\mathrm{Eu}^{3+}$ cryptate, and $5 \mu \mathrm{L}$ of d2-coupled ADP acceptor. ATP hydrolysis to native ADP will compete with d2-coupled ADP, inducing a loss of fluorescence transfert from the anti-ADP cryptate conjugated antibody to the $\mathrm{d} 2$ acceptor. Native ADP formation was thus assessed by measuring the decrease in $\mathrm{d} 2$-coupled ADP fluorescence at $665 \mathrm{~nm}$, reported to the increase in cryptate emission at $620 \mathrm{~nm}$, using a Wallac 1420 VICTOR3 luminometer. Delta F were obtained by calculating $10,000 \times$ the $665 / 625$ ratio, and total ATP conversion was obtained by comparing with Delta F distribution of an ATP/ADP standard curve that mimics the reaction (with total adenosine remaining constant throughout the range).

\section{hHsp70 refolding assay}

Firefly luciferase ( $10 \mu \mathrm{M}$, Sigma-Aldrich) was denatured with $0.2 \mathrm{M}$ guanidinium- $\mathrm{HCl}$ for $30 \mathrm{~min}$ at $25^{\circ} \mathrm{C}$. Refolding of the denatured luciferase $(80 \mathrm{nM})$ at $35^{\circ} \mathrm{C}$ was monitored during $3 \mathrm{~h}$ in the presence of ATP $(1 \mathrm{mM})$ and/or Hsp40 $(160 \mathrm{nM})$ and/or hHsp70 (800 nM) (i.e., the full-length or truncated forms produced by us or from Stressgen). Finally, Dluciferin $(0.25 \mathrm{mM})$ was added, and after $10 \mathrm{~min}$, the percentage of refolded luciferase was measured by collecting total light at $560 \mathrm{~nm}$ by a Wallac 1420 VICTOR $^{3}$ luminometer. The positive control (non-denatured luciferase) was set to $100 \%$. The negative control consists of denatured luciferase in the folding buffer without Hsps. The guanidinium concentration used for the denaturation and the time used for the refolding were optimized for refolding efficiency.

Acknowledgments This work was supported by grants from the Institut National de la Recherche Agronomique and the Burgundy council (PARI Agral 1), and the FEDER (European Funding for Regional Economical Development). F. Neiers thanks the Faber program from the Burgundy region. R. Seigneuric acknowledges a CRCT from section $\mathrm{n}^{\circ} 85$. C. Garrido acknowledges financial support from: the French National Research Agency (ANR) under the program "Investissements d'Avenir" (LabEx ANR-11-LABX-0021), the Institut National du Cancer (INCa), the Ligue Nationale Contre le Cancer ("Label of Excellence"), Ministère de l'Enseignement Supérieur et de le Recherche fellowship, Région Bourgogne, and Université de Bourgogne (BQR). No funding bodies had any role in the study design, data collection and analysis, decision to publish, or preparation of the manuscript. 
Open Access This article is distributed under the terms of the Creative Commons Attribution License which permits any use, distribution, and reproduction in any medium, provided the original author(s) and the source are credited.

\section{References}

Angelidis CE, Lazaridis I, Pagoulatos GN (1999) Aggregation of hsp70 and hsc70 in vivo is distinct and temperature-dependent and their chaperone function is directly related to non-aggregated forms. Eur J Biochem/ FEBS 259:505-512

Aprile FA et al (2013) Hsp70 oligomerization is mediated by an interaction between the interdomain linker and the substrate-binding domain. PLoS One 8:e67961

Benaroudj N, Batelier G, Triniolles F, Ladjimi MM (1995) Selfassociation of the molecular chaperone HSC70. Biochemistry 34: $15282-15290$

Benaroudj N, Triniolles F, Ladjimi MM (1996) Effect of nucleotides, peptides, and unfolded proteins on the self-association of the molecular chaperone HSC70. J Biol Chem 271:18471-18476

Benaroudj N, Fouchaq B, Ladjimi MM (1997) The COOH-terminal peptide binding domain is essential for self-association of the molecular chaperone HSC70. J Biol Chem 272:8744-8751

Bertelsen EB, Chang L, Gestwicki JE, Zuiderweg ER (2009) Solution conformation of wild-type E. coli Hsp70 (DnaK) chaperone complexed with ADP and substrate. Proc Natl Acad Sci U S A 106:8471-8476

Bhattacharya A, Kurochkin AV, Yip GN, Zhang Y, Bertelsen EB, Zuiderweg ER (2009) Allostery in Hsp70 chaperones is transduced by subdomain rotations. J Mol Biol 388:475-490

Blond-Elguindi S, Fourie AM, Sambrook JF, Gething MJ (1993) Peptidedependent stimulation of the ATPase activity of the molecular chaperone $\mathrm{BiP}$ is the result of conversion of oligomers to active monomers. J Biol Chem 268:12730-12735

Boice JA, Hightower LE (1997) A mutational study of the peptidebinding domain of $\mathrm{Hsc} 70$ guided by secondary structure prediction. J Biol Chem 272:24825-24831

Brocchieri L, Conway de Macario E, Macario AJ (2008) hsp70 genes in the human genome: conservation and differentiation patterns predict a wide array of overlapping and specialized functions. BMC Evol Biol 8:19

Brodsky JL, Chiosis G (2006) Hsp70 molecular chaperones: emerging roles in human disease and identification of small molecule modulators. Curr Top Med Chem 6:1215-1225

Bukau B, Deuerling E, Pfund C, Craig EA (2000) Getting newly synthesized proteins into shape. Cell 101:119-122

Calderwood SK (2010) Heat shock proteins in breast cancer progression - a suitable case for treatment? Int J Hyperthermia 26:681-685

Calderwood SK (2013) Molecular cochaperones: tumor growth and cancer treatment. Scientifica 2013:217513

Chappell TG, Konforti BB, Schmid SL, Rothman JE (1987) The ATPase core of a clathrin uncoating protein. J Biol Chem 262:746-751

Dworniczak B, Mirault ME (1987) Structure and expression of a human gene coding for a $71 \mathrm{kd}$ heat shock 'cognate' protein. Nucleic Acids Res 15:5181-5197

Ellis J (1987) Proteins as molecular chaperones. Nature 328:378-379

Ellis DI, Dunn WB, Griffin JL, Allwood JW, Goodacre R (2007) Metabolic fingerprinting as a diagnostic tool. Pharmacogenomics 8: $1243-1266$

Fink AL (1999) Chaperone-mediated protein folding. Physiol Rev 79: 425-449

Flaherty KM, DeLuca-Flaherty C, McKay DB (1990) Three-dimensional structure of the ATPase fragment of a $70 \mathrm{~K}$ heat-shock cognate protein. Nature 346:623-628
Fouchaq B, Benaroudj N, Ebel C, Ladjimi MM (1999) Oligomerization of the 17-kDa peptide-binding domain of the molecular chaperone HSC70. Eur J Biochem / FEBS 259:379-384

Freeman BC, Myers MP, Schumacher R, Morimoto RI (1995) Identification of a regulatory motif in Hsp70 that affects ATPase activity, substrate binding and interaction with HDJ-1. Embo J 14: 2281-2292

Garrido C, Gurbuxani S, Ravagnan L, Kroemer G (2001) Heat shock proteins: endogenous modulators of apoptotic cell death. Biochem Biophys Res Commun 286:433-442

Garrido C, Brunet M, Didelot C, Zermati Y, Schmitt E, Kroemer G (2006) Heat shock proteins 27 and 70: anti-apoptotic proteins with tumorigenic properties. Cell Cycle 5:2592-2601

Gobbo J, Gaucher-Di-Stasio C, Weidmann S, Guzzo J, Garrido C (2011) Quantification of HSP27 and HSP70 molecular chaperone activities. Methods Mol Biol 787:137-143

Gross M, Hessefort S (1996) Purification and characterization of a $66-\mathrm{kDa}$ protein from rabbit reticulocyte lysate which promotes the recycling of hsp 70. J Biol Chem 271:1683316841

Hartl FU, Hayer-Hartl M (2002) Molecular chaperones in the cytosol: from nascent chain to folded protein. Science (New York NY) 295: $1852-1858$

Hartl FU, Hayer-Hartl M (2009) Converging concepts of protein folding in vitro and in vivo. Nat Struct Mol Biol 16:574-581

Hartl FU, Bracher A, Hayer-Hartl M (2011) Molecular chaperones in protein folding and proteostasis. Nature 475:324-332

Hunt C, Morimoto RI (1985) Conserved features of eukaryotic hsp70 genes revealed by comparison with the nucleotide sequence of human hsp70. Proc Natl Acad Sci U S A 82:6455-6459

Jaattela M (1999) Escaping cell death: survival proteins in cancer. Exp Cell Res 248:30-43

Jego G, Hazoume A, Seigneuric R, Garrido C (2010) Targeting heat shock proteins in cancer. Cancer Lett 332:275-285

Jinwal UK et al (2013) Imbalance of Hsp70 family variants fosters tau accumulation. Faseb J 27:1450-1459

Kim D, Lee YJ, Corry PM (1992) Constitutive HSP70: oligomerization and its dependence on ATP binding. J Cell Physiol 153:353-361

Kityk R, Kopp J, Sinning I, Mayer MP (2012) Structure and dynamics of the ATP-bound open conformation of Hsp70 chaperones. Mol Cell 48:863-874

Kumar DP, Vorvis C, Sarbeng EB, Cabra Ledesma VC, Willis JE, Liu Q (2011) The four hydrophobic residues on the Hsp70 inter-domain linker have two distinct roles. J Mol Biol 411: 1099-1113

Macejak D, Rayfield M, Luftig R (1990) Isolation and characterization of human HSP70 expressed in Escherichia coli. Arch Biochem Biophys 280:53-60

Maeda $\mathrm{H}$ et al (2007) Biological heterogeneity of the peptide-binding motif of the $70-\mathrm{kDa}$ heat shock protein by surface plasmon resonance analysis. J Biol Chem 282:26956-26962

Mayer MP, Bukau B (2005) Hsp70 chaperones: cellular functions and molecular mechanism. Cell Mol Life Sci 62:670-684

Nemoto TK, Fukuma Y, Itoh H, Takagi T, Ono T (2006) A disulfide bridge mediated by cysteine 574 is formed in the dimer of the 70 $\mathrm{kDa}$ heat shock protein. J Biochem 139:677-687

Nicolaï A, Delarue P, Senet P (2013) Decipher the mechanisms of protein conformational changes induced by nucleotide binding through free-energy landscape analysis: ATP binding to Hsp70. PLoS Comput Biol 9

Nicoll WS, Boshoff A, Ludewig MH, Hennessy F, Jung M, Blatch GL (2006) Approaches to the isolation and characterization of molecular chaperones. Protein Expr Purif 46:1-15

Palleros DR, Reid KL, Shi L, Fink AL (1993) DnaK ATPase activity revisited. FEBS Lett 336:124-128 
Peake P, Winter N, Britton W (1998) Phosphorylation of Mycobacterium leprae heat-shock 70 protein at threonine 175 alters its substrate binding characteristics. Biochim Biophys Acta 1387:387-394

Powers MV, Workman P (2007) Inhibitors of the heat shock response: biology and pharmacology. FEBS Lett 581:3758-3769

Qi R et al (2013) Allosteric opening of the polypeptide-binding site when an Hsp70 binds ATP. Nat Struct Mol Biol 20:900-907

Rerole AL et al (2011) Peptides and aptamers targeting HSP70: a novel approach for anticancer chemotherapy. Cancer Res 71(2):484-95

Richarme G, Kohiyama M (1993) Autostimulation of the DnaK (HSP 70) ATPase of Escherichia coli. FEBS Lett 322:277-279

Schlecht R, Erbse AH, Bukau B, Mayer MP (2011) Mechanics of Hsp70 chaperones enables differential interaction with client proteins. Nat Struct Mol Biol 18:345-351

Schmid D, Baici A, Gehring H, Christen P (1994) Kinetics of molecular chaperone action. Science (New York NY) 263:971-973

Schmitt E et al (2006) Heat shock protein 70 neutralization exerts potent antitumor effects in animal models of colon cancer and melanoma. Cancer Res 66:4191-4197

Schonfeld HJ, Schmidt D, Schroder H, Bukau B (1995) The DnaK chaperone system of Escherichia coli: quaternary structures and interactions of the DnaK and GrpE components. J Biol Chem 270: 2183-2189

Seigneuric R, Gobbo J, Colas P, Garrido C (2011a) Targeting cancer with peptide aptamers Oncotarget

Seigneuric R, Mjahed H, Gobbo J, Joly A-L, Berthenet K, Shirley S, Garrido C (2011b) Heat shock proteins as danger signals for cancer detection. Front Oncol 1:37
Shi L, Kataoka M, Fink AL (1996) Conformational characterization of DnaK and its complexes by small-angle X-ray scattering. Biochemistry 35:3297-3308

Stangl S et al (2011) Targeting membrane heat-shock protein 70 (Hsp70) on tumors by cmHsp70.1 antibody. Proc Natl Acad Sci U S A 108: 733-738

Swain JF, Dinler G, Sivendran R, Montgomery DL, Stotz M, Gierasch LM (2007) Hsp70 chaperone ligands control domain association via an allosteric mechanism mediated by the interdomain linker. Mol Cell 26:27-39

Taipale M, Jarosz DF, Lindquist S (2010) HSP90 at the hub of protein homeostasis: emerging mechanistic insights. Nat Rev Mol Cell Biol 11:515-528

Thompson AD, Bernard SM, Skiniotis G, Gestwicki JE (2012) Visualization and functional analysis of the oligomeric states of Escherichia coli heat shock protein 70 (Hsp70/DnaK). Cell Stress Chaperones 17:313-327

Venyaminov S, Baikalov IA, Shen ZM, Wu CS, Yang JT (1993) Circular dichroic analysis of denatured proteins: inclusion of denatured proteins in the reference set. Anal Biochem 214:17-24

Vogel M, Mayer MP, Bukau B (2006) Allosteric regulation of Hsp70 chaperones involves a conserved interdomain linker. J Biol Chem 281:38705-38711

Zhang J, Walker GC (1998) Interactions of peptides with DnaK and Cterminal DnaK fragments studied using fluorescent and radioactive peptides. Arch Biochem Biophys 356:177-186

Zhu X, Zhao X, Burkholder WF, Gragerov A, Ogata CM, Gottesman ME, Hendrickson WA (1996) Structural analysis of substrate binding by the molecular chaperone DnaK. Science (New York NY) 272:1606-1614 